\title{
POLARIZATION OF GALACTIC BACKGROUND LIGHT IN THE VISIBLE SPECTRAL REGION
}

\author{
Ch. Leinert \\ Max-Planck-Institut für Astronomie \\ Königstuhl 17, D-6900 Heidelberg FRG
}

\begin{abstract}
This is a summary of the few observations available to date. Although there remain doubts concerning the reliability of most of the published results, a linear polarization of a few percent, perpendicular to the galactic plane, is indicated. Therefore, scattering of galactic light by interstellar dust appears to be the dominating source of galactic background polarization. There is a severe lack of model calculations, which would be needed for a physical interpretation of the data.
\end{abstract}

\section{INTRODUCTION}

Following the general meaning of the words, "galactic background light" includes diffuse galactic light as well as the radiation of distant stars - too faint to be studied individually integrated randomly over a field of at least several arcminutes in size; it excludes reflection nebulae and individual stars or objects like the galactic center. The two components of galactic background light are expected to show different directions of polarization (Figure 1): the diffuse galactic light, resulting from illumination of interstellar dust by the Milky Way as a whole, should show polarization perpendicular to the galactic equator and little dependence on galactic structure; the integrated light of faint stars, subject to interstellar polarization by differential extinction, would be polarized predominantly parallel to the galactic plane and should show a strong dependence on galactic structure, i.e., on the orientation of interstellar magnetic fields and on the distribution of light sources and absorbing matter.

The study of the polarization of galactic background light therefore bears primarily on two points:

(1) To the extent to which it is due to diffuse galactic light, the polarization contains information on the polarizing properties of the scattering dust particles in the general interstellar medium. This particular information cannot be obtained by other means. It complements the information obtained from studies of extinction and polarization of stellar light and of the wavelength dependence of diffuse galactic light brightness.
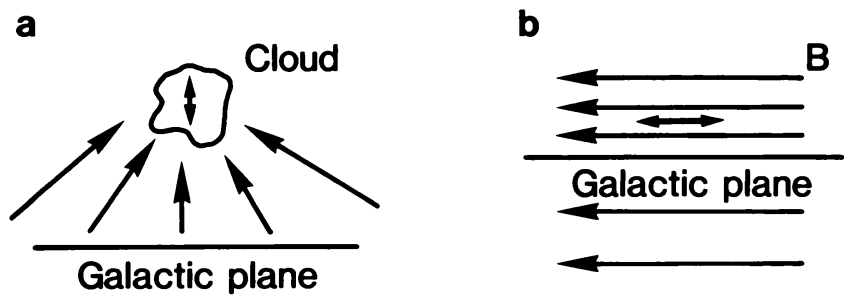

Figure 1. Expected direction of polarization for (a) scattered light and (b) transmitted light polarized by differential extinction. The case of scattered light has been illustrated for a high-latitude cloud; however, the same direction of polarization also results for a cloud in the galactic plane, because the geometry of illumination basically is the same. Differential extinction results in polarization parallel to the interstellar magnetic field, $B$. 
(2) Mainly because of its component of integrated starlight, the polarization contains information on the galactic structure, by which it is determined in the first place. A sufficiently complete model of the galaxy will allow the prediction of the polarization of galactic background light; the model can then be tested by measurements of this polarization. Such constraints can then be incorporated in models of the galaxy.

Ideally, the two effects would be observed separately. In practice, they are usually measured together. A small size (arcmininute) field-of-view and carefully selected viewing directions are necessary to achieve or at least approach separation of the components.

To my knowledge there are very few observations of polarization of galactic background light reported in the literature (see Figure 2). This field has almost been forgotten. One purpose of this review, therefore, is to bring the existence of this field to the attention of a wider audience and perhaps to focus new interest on it. The following sections will discuss the difficulties of the measurements, which are particularly pronounced for ground-based studies; summarize the few available, partly contradictory results; and demonstrate the lack of theoretical studies.

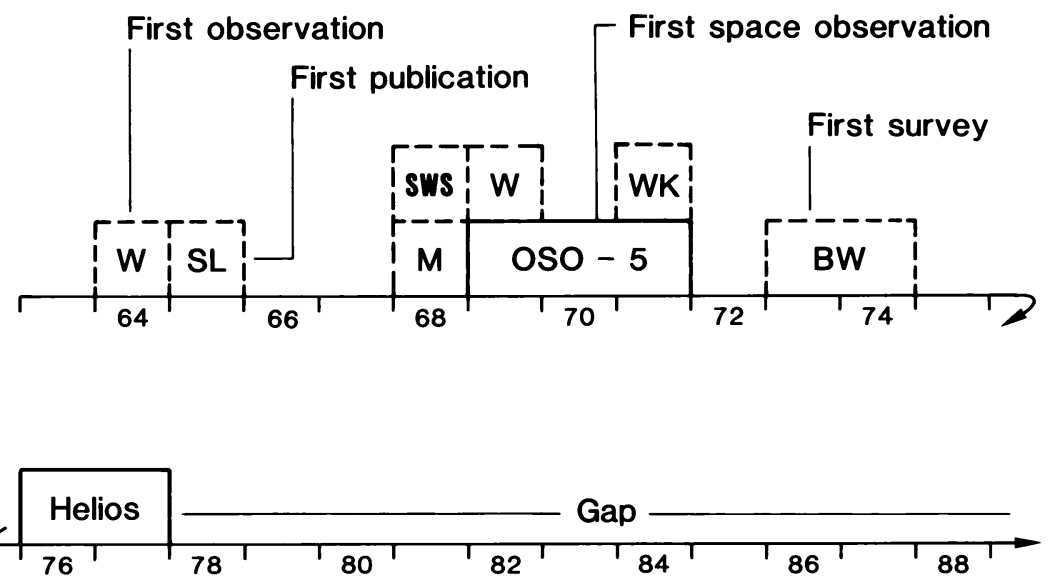

Figure 2. Summary of observations. For identification of authors, refer to Table 1.

\section{FOREGROUND POLARIZATION}

There are two sources of foreground polarization which have to be corrected for, atmospherically scattered light and zodiacal light. While the first one can be avoided by observing from above the troposphere, the second one is always important. Only a spacecraft in the outer solar system would not have to worry about foreground polarization, but such measurements have not yet been performed. The airglow continuum is not expected to be polarized.

\subsection{Zodiacal Light}

The polarization due to zodiacal light is large compared with that due to the galactic background light. Even in the antisolar hemisphere, the polarized intensity may reach $2.5 \times 10^{-7} \mathrm{~W} \mathrm{~m}^{-2} \mathrm{sr}^{-1} \mu \mathrm{m}^{-1}$, one order of magnitude more than the signal to be measured, although zodiacal light polarization should be small within $15^{\circ}$ around the antisolar point. Published zodiacal light data have too large an uncertainty $(\Delta p= \pm 0.01-0.02)$ to be used for 
TABLE 1. Available Observations of Galactic Background Polarization

\begin{tabular}{|c|c|c|c|c|c|}
\hline Authors & $\begin{array}{c}\text { Wavelength } \\
(\mathrm{nm})\end{array}$ & Observatory & $\begin{array}{c}\text { Period of } \\
\text { Observations }\end{array}$ & $\begin{array}{c}\text { Spatial } \\
\text { Coverage } \\
\text { (sr) }\end{array}$ & Result \\
\hline Wolstencroft (1973) & 708 & $\begin{array}{l}\text { Chacaltaya } \\
5200 \mathrm{~m}\end{array}$ & Aug 1964 & 0.4 & $\begin{array}{l}p l>10 S_{10}(R) \\
\text { at } l=90^{\circ}, 270^{\circ}\end{array}$ \\
\hline $\begin{array}{l}\text { Schmidt and Leinert } \\
\text { (1966) }\end{array}$ & $400-600$ & $\begin{array}{l}\text { Königstuhl } \\
525 \mathrm{~m}\end{array}$ & Sept 1965 & $2 \times 10^{-5}$ & $\begin{array}{l}p=2.2 \pm 0.4 \%, \\
\text { different from stars }\end{array}$ \\
\hline Mattila (1970) & $400-600$ & $\begin{array}{l}\text { Boyden } \\
1400 \mathrm{~m}\end{array}$ & May 1968 & $1 \times 10^{-5}$ & $\begin{array}{l}p=1.7 \pm 0.7 \% \\
\text { tgal. equator }\end{array}$ \\
\hline $\begin{array}{l}\text { Wolstencroft and Kemp } \\
\text { (1972) }\end{array}$ & $450-650$ & $\begin{array}{l}\text { Mauna Kea } \\
4100 \mathrm{~m}\end{array}$ & $1971(?)$ & $10^{-6}$ & $\begin{array}{l}\text { circular polarization } \\
q=-0.58 \pm 0.14 \%\end{array}$ \\
\hline Staude et al. (1973) & 420 and 526 & Boyden & May-Dec 1968 & 0.4 & $\begin{array}{l}p \approx 2 \% \text {, I gal. equator, } \\
\text { circular polarization detected }\end{array}$ \\
\hline Wolstencroft (1973) & 508 & $\begin{array}{l}\text { Haleakala } \\
3100 \mathrm{~m}\end{array}$ & Jan 1969 & 0.2 & $\begin{array}{l}p I \approx 8 S_{10}(V), \| \text { gal. } \\
\text { equator, preliminary }\end{array}$ \\
\hline $\begin{array}{l}\text { Bandermann and } \\
\text { Wolstencroft (1976) }\end{array}$ & 508 & $\begin{array}{l}\text { Haleakala } \\
3100 \mathrm{~m}\end{array}$ & $\begin{array}{l}\text { Apr } 1969 \\
\text { May } 1971\end{array}$ & 4 & night sky data only \\
\hline Sparrow and Ney (1972) & $B$ and $R$ & $\begin{array}{l}\text { OSO-5 } \\
\text { satellite }\end{array}$ & Mar 1969 and 1971 & 0.07 & $\begin{array}{l}p I \approx 4 S_{10}(B) \\
\text { if } \downarrow \text { gal. equator } \\
p I \approx 18 S_{10}(R)\end{array}$ \\
\hline $\begin{array}{l}\text { Bandermann and } \\
\text { Wolstencroft (1976) }\end{array}$ & 530 & $\begin{array}{l}\text { Haleakala } \\
3100 \mathrm{~m}\end{array}$ & $\begin{array}{l}\text { May } 1973 \\
\text { Nov } 1974\end{array}$ & $\begin{array}{c}4.5 \\
+ \text { gal. equator }\end{array}$ & $p I=4-10 S_{10}(V)$ \\
\hline $\begin{array}{l}\text { Leinert and Richter } \\
\text { (1983) }\end{array}$ & 530 & $\begin{array}{l}\text { Helios } 1,2 \\
\text { space probes }\end{array}$ & $\begin{array}{l}\text { Oct 1975-Feb } 1976 \\
\text { Nov 1976-Mar } 1977\end{array}$ & 0.02 & $\begin{array}{l}p I=1-2 S_{10}(V) \\
+ \text { gal. equator }\end{array}$ \\
\hline
\end{tabular}

Transformation of $S_{10}$ units to physical quantities is as follows:

$B, 420 \mathrm{~nm}-1 S_{10}=2.2 \times 10^{-8} \mathrm{~W} \mathrm{~m}^{-2} \mathrm{sr}^{-1} \mu \mathrm{m}^{-1} \lambda \mathrm{F}_{\lambda}=9.2 \times 10^{-9} \mathrm{~W} \mathrm{~m}^{-2} \mathrm{sr}^{-1}$

$V, 530 \mathrm{~nm}-1 S_{10}=1.4 \times 10^{-8} \mathrm{~W} \mathrm{~m}^{-2} \mathrm{sr}^{-1} \mu \mathrm{m}^{-1} \lambda \mathrm{F}_{\lambda}=7.4 \times 10^{-9} \mathrm{~W} \mathrm{~m}^{-2} \mathrm{sr}^{-1}$

$R, 700 \mathrm{~nm}-1 S_{10}=5.6 \times 10^{-9} \mathrm{~W} \mathrm{~m}^{-2} \mathrm{sr}^{-1} \mu \mathrm{m}^{-1} \lambda \mathrm{F}_{\lambda}=3.9 \times 10^{-9} \mathrm{~W} \mathrm{~m}^{-2} \mathrm{sr}^{-1}$

the removal of foreground polarization. The correction for zodiacal light polarization therefore has to be deduced from the same data set that gives the polarization of galactic background light.

Observations from the Helios space probes have shown the zodiacal light intensity to be remarkably stable, within $2 \%$ over a decade (Leinert and Pitz, 1989). However, seasonal variations exist because of the motion of the observer with respect to the cloud of interplanetary dust and its plane of symmetry, which deviates from the ecliptic by a few degrees (Dumont and Levasseur-Regourd, 1978; Leinert et al., 1980). These variations, which appear as well in polarized light, amount to a few percent for viewing directions close to the ecliptic, but they may reach $\pm 10 \%$ at the ecliptic poles and have to be given proper attention in the data reduction.

\subsection{Atmospherically Scattered Light}

This component of polarized light of the night sky depends on the brightness of the airglow as well as on the position of Milky Way and zodiacal light on the sky and relative to the viewing direction. Since this effect is difficult to measure, detailed corrections have to rely on scattering calculations. The most comprehensive ones are those of Staude (1975) for first order Rayleigh and Mie scattering in the Earth's atmosphere. Stande gives results for a sufficient number of different optical depths and different positions of Milky Way and zodiacal light to allow interpolation to virtually all conditions occurring in practice. The effect of higher-order 
scattering is included by means of semi-empirical correction factors. Earlier calculations by Wolstencroft and van Breda (1967) covered only one specific case and used a model of zodiacal light that no longer can be considered representative.

Figure 3 shows the resulting calculated polarization of atmospherically scattered light for a situation, where the Milky Way is in the zenith, the galactic center is near the horizon, and the solar depression is $16^{\circ}$. The effect is comparable in size with the galactic background polarization. Although the slow variation with zenith distance may help to correct for it, it also makes it hard to recognize the actual amount of atmospheric polarization present in a data set. It is an advantage of space observations that they are free of this effect.
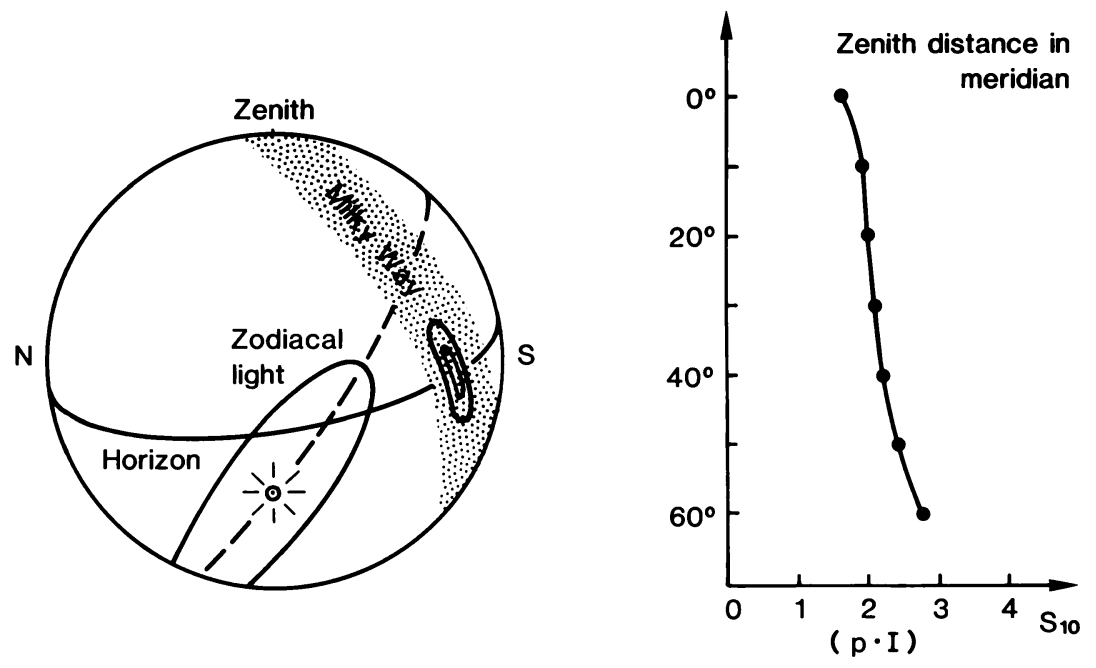

Figure 3. Polarized intensity of atmospherically scattered light along the meridian for the geometry shown in the left part of the figure (According to Staude, 1975).

\section{OBSERVATIONS}

The record is scarce. The overview in Figure 2 shows only nine published observations, about which some relevant information is summarized in Table 1 . All the observations fall into the period 1964-1977, and none happened during the last decade. This fact demonstrates that these measurements are considered difficult, or unrewarding, or both. Section 4 looks into the relevance of galactic background polarization measurements. The degree of difficulty involved will be apparent from the following discussion.

\subsection{Method}

As stressed above, galactic background light always has to be observed in the presence of the strong foreground of zodiacal light, which in general is highly polarized. The only experiment essentially free of zodiacal light contamination was the photopolarimeter on Pioneer 10/11 (Weinberg, 1981) during its measurements at positions beyond 3 AU. Unfortunately, the experiment designed for the study of Jupiter's bright and highly polarized atmosphere did not produce quality polarization measurements of galactic background polarization. Hence, we are left with the task of disentangling the sum of the galactic and zodiacal surface polarization. Expressing the polarization with the help of Stokes' parameters $Q$ and $U$, the observed values 
may be written as

$$
\begin{aligned}
& Q_{o b s}\left(b, l, \beta, \lambda-\lambda_{\odot}\right)=Q_{\text {gal }}(b, l)+Q_{\text {zod }}\left(\beta, \lambda-\lambda_{\odot}\right) \\
& U_{o b s}\left(b, l, \beta, \lambda-\lambda_{\odot}\right)=U_{g a l}(b, l)+U_{\text {zod }}\left(\beta, \lambda-\lambda_{\odot}\right)
\end{aligned}
$$

for observations above the atmosphere, where $b, l$ are galactic and $\beta, \lambda-\lambda_{\odot}$ helioecliptic coordinates. For earthbound observations, the extinction of extraterrestrial radiation and an additional brightness due to tropospheric scattering add complexity to the relation:

$$
\begin{gathered}
Q_{o b s}=\left[Q_{g a l}+Q_{z o d}\right] e^{-\tau}+Q_{s c a} \\
U_{o b s}=\left[U_{g a l}+U_{z o d}\right] e^{-\tau}+U_{s c a} .
\end{gathered}
$$

So far, the contribution of tropospheric scattered light has not explicitly been corrected for in ground-based observations. Several methods have been used to separate the components in equations (1) and (2).

3.1.1. Differential measurements. This method was applied by Mattila (1970) to a highlatitude dark cloud in Libra. It appears safe to assume that nearby $\left(\leq 1^{\circ}\right)$ reference points outside the cloud have the same nongalactic foreground polarization as the measured field. Also since the contribution of brighter stars to these fields can be excluded by careful selection of the field-of-view, this method should be reliable. However, it is restricted to a few suitable fields.

The other methods mostly use the relative seasonal shift of the zodiacal light and the Milky Way to perform the separation. They rest on the assumption that certain reference points (e.g., at high galactic latitudes, $|b|>30^{\circ}$ ) do not have measurable polarization of their galactic background. Adequate subtraction of the Stokes' parameters measured in such a reference field from equations (1) and (2), respectively, cancels the unwanted terms. Four such schemes have been used so far, three of which are shown in Figure 4.

3.1.2. Symmetrical points (Figure 4a). One assumes that the zodiacal light polarization is symmetrical with respect to the ecliptic. The reference position is chosen to be at $\left(-\beta, \lambda-\lambda_{\odot}\right)$ and at high galactic latitude. The reference position is observed as closely at the same time as possible (Wolstencroft, 1973). (However, on satellite OSO-5, this time difference amounted to several months.) It should be noted that the assumed symmetry is only approximate, because of the non-ecliptic symmetry of the interplanetary dust distribution.

3.1.3. Two epochs (Figure $4 b$ ). One assumes that zodiacal light polarization is stable. The measurement at position $\left(\beta, \lambda-\lambda_{\odot}\right)$ is repeated at another time, when it has high galactic latitude. Preferably, measurements at various epochs are used to construct the average zodiacal light polarization at $\left(\beta, \lambda-\lambda_{\odot}\right)$, which then is subtracted directly from equations (1) or (2) (Bandermann and Wolstencroft, 1976). Again the assumption is not strictly fulfilled, because of the seasonal variations mentioned above.

3.1.4. Spatial interpolation. A smooth surface is fitted through a grid of reference observations at high galactic latitudes to estimate the nongalactic sky polarization at low galactic latitudes (Staude, Wolf, and Schmidt, 1973). The accuracy depends on how well the fit corresponds to reality. The results are not encouraging.

3.1.5. Temporal interpolation (Figure $4 c)$. One assumes that $Q_{z o d}\left(\beta, \lambda-\lambda_{\odot}\right), U_{z o d}\left(\beta, \lambda-\lambda_{\odot}\right)$ vary smoothly enough with heliocentric position of the observer to allow an interpolation to the time when the field $\left(\beta, \lambda-\lambda_{\odot}\right)$ crosses the galactic plane. This method requires months of stable operation under unchanging conditions and, therefore, is reserved to space experiments. It also can be applied to the difference between "symmetrical points" (see 3.1.2, above). The validity 

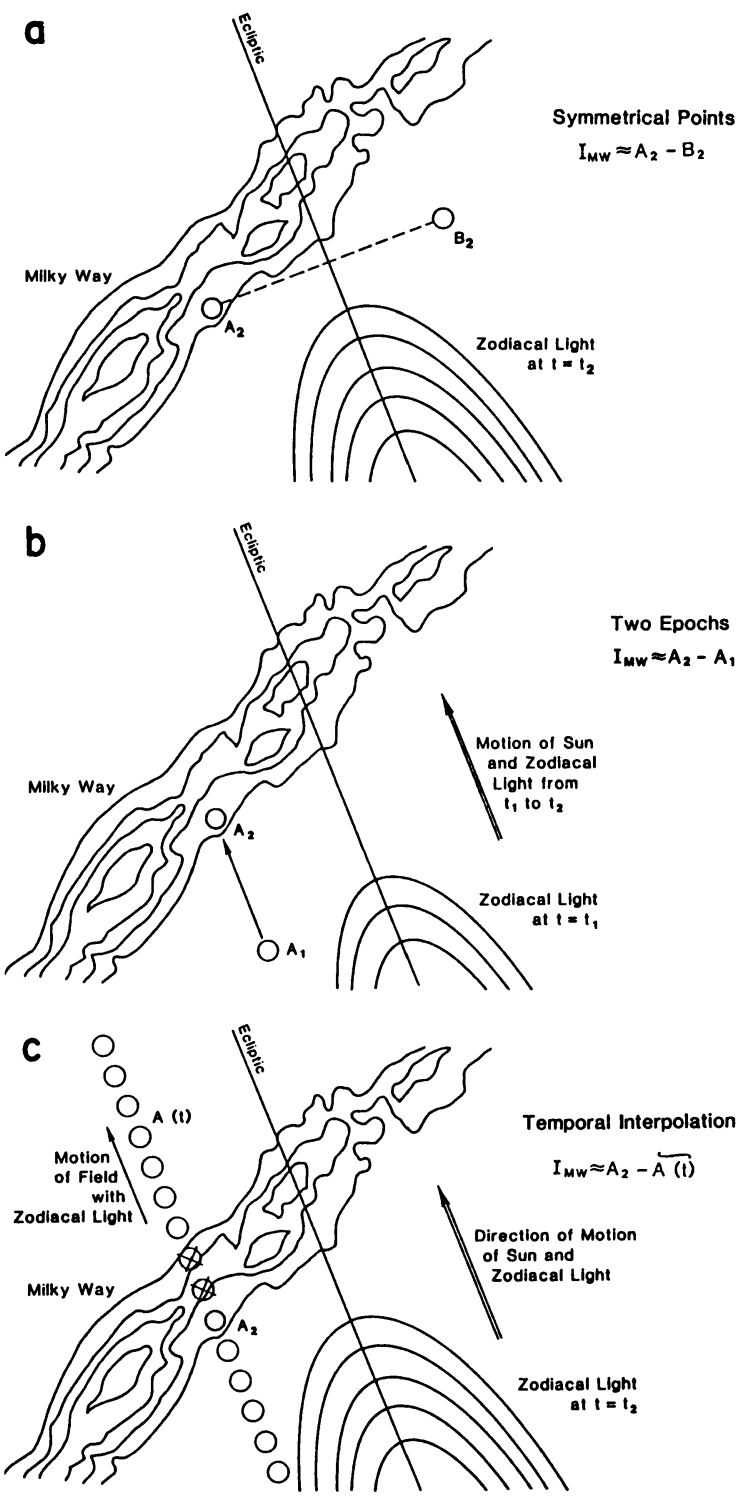

Figure 4. Three methods to subtract the foreground polarization from measurements taken at point $\mathrm{A} 2$ at time $t_{2}$ (see text). $\widetilde{A(t)}$ is the interpolation of measured polarization $A(t)$ for the time $t_{2}$. Crosses indicate that those measurements are not used in the definition of the interpolation. validity of the assumption may be judged from the data shown in Figure 5 (Leinert and Richter, 1983).

Among these five methods, those in sections 3.1.1 and 3.1.5 are the most differential measurements and therefore are to be preferred.

\subsection{Results}

The most complete coverage of the Milky Way is given by the work of Bandermann and Wolstencroft (1976, Figure 6). To judge its reliability, an intercomparison with the other available data is helpful. As shown in Figure 6 and in Table 2, there is a striking degree of inconsistency and even contradiction. This, of course, casts doubt upon all the measurements involved. The standard excuse, that the object polarization might be variable, is not applicable in the case of the Milky Way. A more plausible explanation would be that correction for the effects of atmospheric scattered light have not been properly performed in all of the older groundbased experiments. Clearly, space experiments would be preferable, but so far they give only insufficient coverage.

In this situation any comments on the observed results are highly subjective. Nevertheless, in order to provide a guideline, I suggest the following critical summary:

- Given the long-term stability of the zodiacal light experiment on Helios, proven by the repeatability of measurements over one full solar cycle (Leinert and Pitz, 1989), the results of this space probe experiment should be given great weight. Both at $l=150^{\circ}$ and $l=223^{\circ}$, the degree of polarization observed around $b=0^{\circ}$ was $p=1.1 \pm 0.2 \%$.

- Mattila's (1970) measurements refer to a special differential geometry and are less sensitive to systematic errors. His result of $p=1.7 \pm$ $0.2 \%$ should be reliable for the 


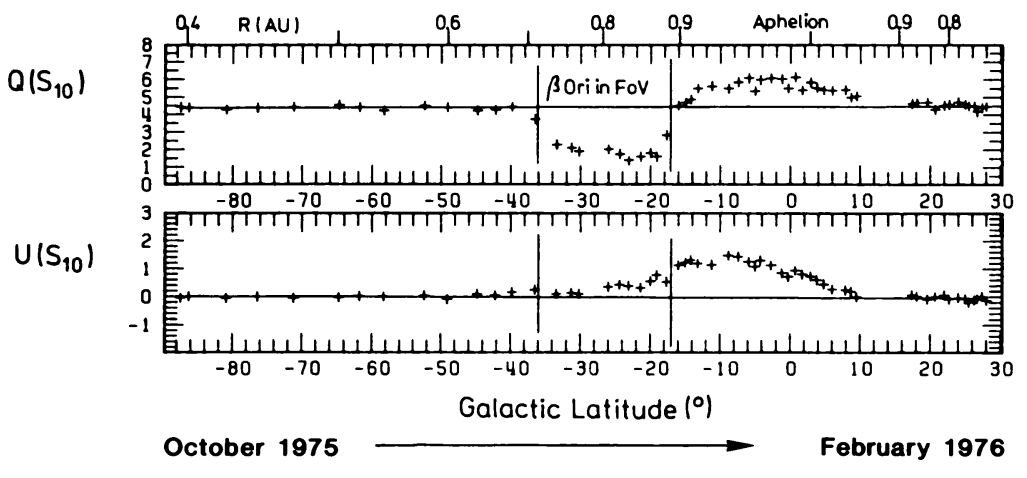

Figure 5. Milky Way polarization measured by Helios 1 at galactic longitude $l=220^{\circ}$ according to the method of temporal interpolation. The polarization is given in ecliptic coordinates. It corresponds to polarization of $p I=1.8 \pm 0.3 S_{10}(V)$ almost perpendicular to the galactic equator. Each value is the average over a $19^{\circ} \times 2^{\circ}$ field at ecliptic latitude $\beta=-31^{\circ}$.

TABLE 2. Intercomparison of Measured Polarization Vectors of Galactic Background Polarization

\begin{tabular}{|c|c|c|c|c|}
\hline \multirow[b]{2}{*}{$\begin{array}{l}\text { Author } \\
(l, b)\end{array}$} & \multirow[b]{2}{*}{ Measured polarization } & \multirow[b]{2}{*}{$\begin{array}{l}\text { Value given by Bandermann } \\
\text { and Wolstencroft }\end{array}$} & \multicolumn{2}{|c|}{ Difference } \\
\hline & & & Absolute & $\begin{array}{l}\text { Relative } \\
\text { to } \sigma\end{array}$ \\
\hline $\begin{array}{l}\text { Schmidt and Leinert } \\
\left(101^{\circ},-1.5^{\circ}\right)\end{array}$ & $p=2.1 \%, \theta_{g}=103^{\circ}$ & $p I=5.6 \pm 1.3 S_{10}(V), \theta_{g} \approx 15^{\circ}$ & $p l \approx 8.2 S_{10}$ & 6.3 \\
\hline $\begin{array}{l}\text { Staude et al. } \\
\left(0^{\circ}, 0^{\circ}\right)\end{array}$ & $p=2 \%, \theta_{g} \approx 90^{\circ}$ & $p I \approx 8.9 \pm 0.8 S_{10}(V), \theta_{g} \approx 26^{\circ}$ & $p l \approx 15 S_{10}$ & 11.5 \\
\hline $\begin{array}{l}\text { Wolstencroft, } 508 \mathrm{~nm} \\
\left(165^{\circ},+8^{\circ}\right)\end{array}$ & $p I=11 S_{10}, \theta_{g} \approx 85^{\circ}$ & $p I \approx 4.4 \pm 0.8 S_{10}(V), \theta_{g}=6^{\circ}$ & $p I \approx 15 S_{10}$ & 6.8 \\
\hline $\begin{array}{r}\text { Helios }\left(150^{\circ}, 10^{\circ}\right) \\
\left(220^{\circ},-5^{\circ}\right)\end{array}$ & $\begin{array}{l}p I=1.1 S_{10}, \theta_{g}=-4^{\circ} \\
p I=1.8 S_{10}, \theta_{g}=+11^{\circ}\end{array}$ & $\begin{array}{l}p l \approx 3.9 \pm 0.6 S_{10}(V), \theta_{g}=-11^{\circ} \\
p l \approx 6.9 \pm 1.1 S_{10}(V), \theta_{g}=27^{\circ}\end{array}$ & $\begin{array}{l}p l \approx 3.0 S_{10} \\
p l \approx 5.5 S_{10}\end{array}$ & $\begin{array}{l}4.5 \\
4.8\end{array}$ \\
\hline
\end{tabular}

$\theta_{g}$ is measured counterclockwise on the sky; $\theta_{g}=0$ gives the direction to the North Galactic Pole.

particular region he studied.

- The experiment on OSO-5 indicated a galactic background polarization perpendicular to the galactic plane. However, the deduced amount of polarization depends on the assumed direction of polarization and may have been influenced by the seasonal variation of zodiacal light. Therefore, its quantitative results should be viewed with caution.

- The results of Bandermann and Wolstencroft, based on a one-year average, should be qualitatively correct. Quantitatively, the large differences evidenced in Table 2 suggest that the actual uncertainty must be considerable. Probably the values are high, an extreme case being the polarized intensities of $p I>10 S_{10}(V)$ near the north ecliptic pole. (Their results derive from the same data set that yielded non-zero zodiacal light polarization at the antisolar point and night-to-night changes of zodiacal light polarization [Bandermann and Wolstencroft, 1974], both unconfirmed or challenged [Sparrow and Weinberg, 1975; Leinert and Planck, 1982].) 


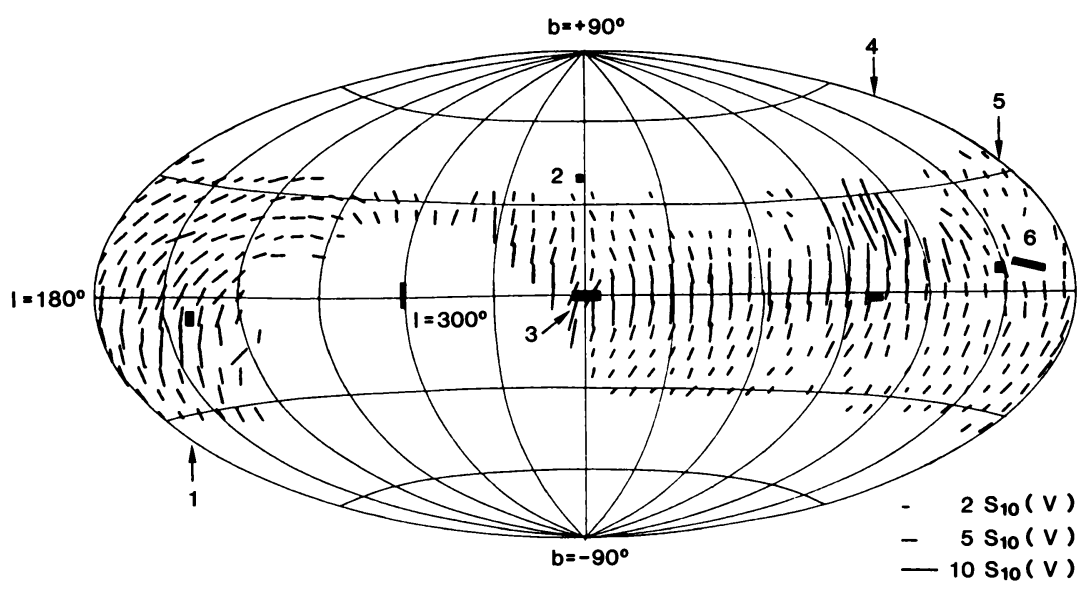

Figure 6. Milky Way polarization according to Bandermann and Wolstencroft (1976). The vectors give polarized intensity, the direction is as seen from outside the celestial sphere. For comparison, measurements by Helios (1, 5), Mattila (2), Staude et al. (3), Schmidt and Leinert (4) and Wolstencroft (6) are added to their diagram.

- The remaining results should be confirmed by new measurements before being accepted as real: some of them are incompatible with the results of Bandermann and Wolstencroft.

In total, little more can be said from the measurements on the polarization of galactic background light than that it appears to be oriented perpendicular to the galactic plane with a degree of polarization of typically $1-2 \%$.

In view of the difficulties already apparent in the measurement of linear polarization, it seems premature to discuss the reports on circular polarization of galactic background light (Staude, Wolf, and Schmidt, 1973; Wolstencroft and Kemp, 1972), although a confirmed effect would be a quite specific indicator of particle properties.

\section{INTERPRETATION}

For the interpretation of galactic background polarization, it would be helpful to have separated the effects of scattering and interstellar polarization by differential extinction. Among the present measurements, it may be assumed only for Mattila's (1970) observations in a dark cloud that one effect - in this case, scattering - clearly is dominating.

\subsection{Polarization of Diffuse Galactic Light}

If $S_{1}(\theta), S_{2}(\theta)$ are the average scattering functions of diffuse interstellar dust for scattering light polarized perpendicular and parallel, respectively, to the scattering plane (average over the ensemble of particles), then the observed degree of polarization for single scattering is

$$
p=\frac{<S_{1}(\theta)-S_{2}(\theta)>}{<S_{1}(\theta)+S_{2}(\theta)>}
$$


where the average $<>$ is a brightness-weighted mean over all scattering angles occurring with the Milky Way as light source. We obtain from the measurements some average over the polarizing properties of interstellar dust for large-angle scattering. As mentioned above, this contains information not obtainable by the study of interstellar reddening, interstellar polarization, or wavelength dependence of diffuse galactic light. However, this information cannot be exploited without resort to realistic model calculations. Therefore, the most obvious conclusion of this review would be that a grid of such model calculations is needed. This would allow us to demonstrate the degree to which measurements of the polarization of galactic background light are able to discriminate particle properties, and it would form a basis on which to plan future observations.

So far only one short exploratory calculation is available (Mattila 1971, unpublished). For the geometry of a cloud in the galactic plane (Figure 7), he calculated that the resulting polarization of scattered light should be $p=17 \%$ for Rayleigh particles, or $p=6 \%$ for submicron ice particles. Hence the existing observations, which give lower values of $p=1-2 \%$, already provide useful constraints and indicate the value of this field of study.
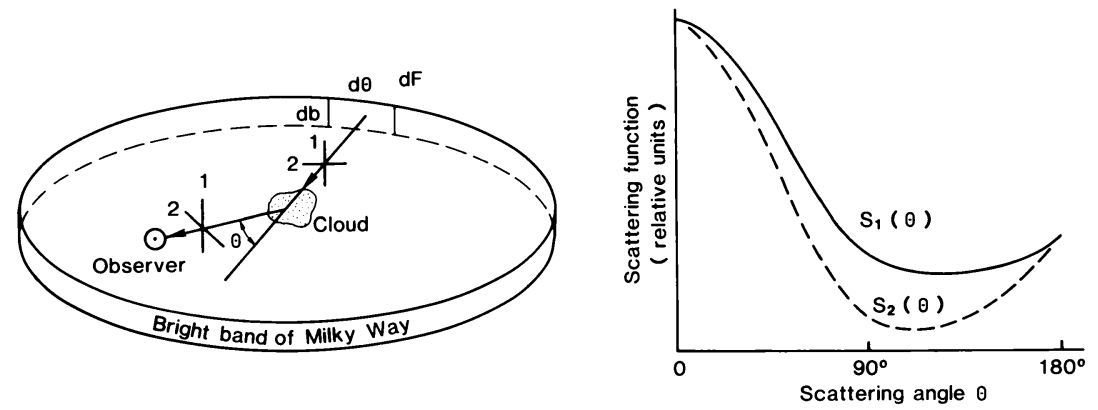

Figure 7. Scattering geometry and scattering function (submicron ice particles) used by Mattila (1971) to predict the polarization of scattered light in a dark cloud.

\subsection{Polarization of Galactic Background Light by Differential Extinction}

A difficulty with the interpretation of such observations lies in the fact that the polarizing dust particles inevitably also scatter the light incident from all directions. Provided the fraction of polarization due to scattering can be separated, the polarization of transmitted galactic background light has the advantage that it refers to larger distances than do measurements of interstellar polarization on individual stars. The geometry is more complicated than in the case of extragalactic sources seen face-on (Scarrott, Ward-Thompson, and Warren-Smith, 1987). But one may expect to get information on the structure of magnetic field and distribution of dust and light sources at larger distances, perhaps by techniques like that used by Lloyd and Harwit (1973) to localize polarizing regions from stellar polarization measurements. In any case, regardless of whether a decomposition according to polarizing effects is possible, the measurements of polarization of galactic background light contribute an additional test on the applicability of detailed galactic models. 


\section{CONCLUSION}

Despite the difficulties of measurement and interpretation, the polarization of galactic background light may be expected to contribute worthwhile new information on interstellar dust and galactic structure. To this end, new work in this field is needed, especially in the area of model calculations.

Acknowledgment - I thank Kalevi Mattila for helpful comments.

\section{REFERENCES}

Bandermann, L. W., and Wolstencroft, R. D. 1974, Nature, 252, 215.

Bandermann, L. W., and Wolstencroft, R. D. 1976, Mon. Not. R. Astr. Soc., 81, 37.

Dumont, R., and Levasseur-Regourd, A.-Ch. 1978, Astron. Astrophys., 64, 9.

Leinert, Ch., Hanner, M., Richter, I., and Pitz, E. 1980, Astron. Astrophys., 82, 328.

Leinert, Ch., and Planck, B. 1982, Astron. Astrophys., 105, 364.

Leinert, Ch., and Richter, I. 1983, Astron. Astrophys., 121, 146.

Leinert, Ch., and Pitz, E. 1989, Astron. Astrophys., 210, 399.

Lloyd, St., and Harwit, M. 1973, in Interstellar Dust and Related Topics, ed. M. Greenberg and H. C. Van de Hulst, IAU Symposium 52, Reidel, Dordrecht, 203.

Mattila, K. 1970, Astron. Astrophys., 8, 273.

Mattila, K. 1971, unpublished report.

Scarrott, S. M., Ward-Thompson, D., and Warren-Smith, R. F. 1987, Mon. Not. R. Astr. Soc., 224, 299.

Schmidt, Th., and Leinert, Ch. 1966, Z.f. Astrophys., 64, 110.

Sparrow, J. G., and Ney, E. P. 1972, Astrophys. J., 174, 717.

Sparrow, J. G., and Weinberg, J. L. 1975, Astron. Astrophys., 41, 475.

Staude, H. J. 1975, Astron. Astrophys., 39, 325.

Staude, J., Wolf, K., and Schmidt, Th. 1973, in Interstellar Dust and Related Topics, ed. M. Greenberg and H. C. Van de Hulst, IAU Symposium 52, Reidel, Dordrecht, 139.

Weinberg, J. L. 1981, Sky and Telescope, 61, 114.

Wolstencroft, R. D., and van Breda, I.G. 1967, Astrophys. J. 147, 255.

Wolstencroft, R. D., and Kemp, I. C. 1972, Astrophys. J. (Letters), 177, L137.

Wolstencroft, R. D. 1973, in Interstellar Dust and Related Topics, ed. M. Greenberg and H. C. Van de Hulst, IAU Symposium No. 52, Reidel, Dordrecht, 99.

H. Okuda: In studies of the galactic magnetic field, polarization measurements of infrared sources are very useful due to the good transparency of interstellar space at infrared wavelengths. In fact, we have made such a measurement in the galactic center region and found that the observed polarizations are fairly regular, mostly parallel to the galactic plane, indicating that the magnetic field runs in the galactic plane (Okuda, IAU Symposium No. 106, "The Milky Way Galaxy").

F. Paresce: How do you explain the low values (1-2\%) of polarization observed? Would you not expect it to be higher (15-20\%) for typical scattering particles (Rayleigh scatterers) and angles?

Ch. Leinert: Several effects may contribute to this. First, the superposition of interstellar polarization by differential extinction, which is polarized at $90^{\circ}$ to the scattered light polarization Second, Prepolarization of the illuminating light source, the Milky Way, itself - by interstellar polarization. Third, Rayleigh particles may not be really typical. Model calculations are needed to predict expected polarization as function of particle material for probable size distributions. 